\title{
The examination of some anthropometric and motoric characteristics of students aged eight years according to their maturation level
}

Authors' Contribution: A Study Design B Data Collection C Statistical Analysis D Data Interpretation E Manuscript Preparation F Literature Search G Funds Collection

\author{
Erdal Ari ${ }^{1} \mathrm{ABCD}$, Gökhan Deliceoglu2 $\mathrm{ABCD}$ \\ ${ }^{1}$ Ordu University Physical Education and Sports School, Turkey \\ ${ }^{2}$ Kırıkkale University Faculty of Sports Sciences, Kırıkkale, Turkey
}

\section{abstract}

Background: Maturation is an important factor for exercise. The maturation effect on some anthropometric and motoric characteristics of students aged eight years was examined in this study.

Material and methods:

535 primary education students voluntarily participated in this study. Anthropometric measurements (weight, height, sitting height, stroke length, stroke rate, leg length and body mass index), flexibility, handgrip strength, vertical jump (squat and countermovement jump), tapping (skipping) test paramaters (step frequency, flight times and contact times to ground of right and left foot, step/speed ratio), postural sway (anterior-posterior, medial lateral and total postural sway), visual reaction and reaction time, 20 meters sprint time, agility values were measured. The test parameters were compared in terms of gender and maturation level (early, normal and delayed maturation) by parametric (independent samples T-test, one way ANOVA) and non-parametric (Mann Whitney-U and Kruskal Wallis-H) tests.

Results: A significant difference was indicated between boy and girl students in most of the test parameters $(p<0.05)$. The analysis results indicated that students at early maturation level had better test values $(p<0.05)$.

Conclusions: The maturation factor positively affected some anthropometric and motoric parameters of students aged eight years.

Key words: maturation, maturation offset, anthropometric measurements, students, children.

\section{article details}

Article statistics: Word count: 7,482; Tables: 10; Figures: 1; References: 37

Received: March 2020; Accepted: April 2020; Published: June 2020

Full-text PDF: http://www.balticsportscience.com

Copyright Indexation: (c) Gdansk University of Physical Education and Sport, Poland

Celdes, Clarivate Analytics Emerging Sources Citation Index (ESCI), CNKI Scholar (China National Knowledge Infrastructure), CNPIEC, De Gruyter - IBR (International Bibliography of Reviews of Scholarly Literature in the Humanities and Social Sciences), De Gruyter - IBZ (International Bibliography of Periodical Literature in the Humanities and Social Sciences), DOAJ, EBSCO - Central \& Eastern European Academic Source, EBSCO - SPORTDiscus, EBSCO Discovery Service, Google Scholar, Index Copernicus, J-Gate, Naviga (Softweco, Primo Central (ExLibris), ProQuest - Family Health, ProQuest - Health \& Medical Complete, ProQuest - Illustrata: Health Sciences, ProQuest - Nursing \& Allied Health Source, Summon (Serials Solutions/ProQuest, TDOne (TDNet), Ulrich's Periodicals Directory/ulrichsweb, WorldCat (OCLC)

Funding: This research received no specific grant from any funding agency in the public, commercial, or not-for-profit sectors.

Conflict of interests: Corresponding author: Open Access License:
Authors have declared that no competing interest exists.

Erdal ARI, Ordu University Physical Education and Sports School Ordu, TURKEY, arierdal@hotmail.com.

This is an open access article distributed under the terms of the Creative Commons Attribution-Non-commercial 4.0 International (http://creativecommons.org/licenses/by-nc/4.0/), which permits use, distribution, and reproduction in any medium, provided the original work is properly cited, the use is non-commercial and is otherwise in compliance with the license. 


\section{INTRODUCTION}

Maturation is a consequence of physical growth, and it can be defined as a functional development of limbs. The development of tissues, organs and systems in the body is known as biological maturation [1]. The development of endocrin and nervous system in growth and maturation period is effective on skeletal and physical maturation [2]. Malina and Kozıeł [3] indicated that biological maturation could be examined with chronological age and maturation timing factors. The chronological age is a indicator of human growth and it can be equal to the real maturation level. The biological maturation level of children in same chronological age may widely differ [4, 5]. Sometimes, children's real maturation levels can be higher than the maturation level of chronological age. The body systems, physical and psychological features of individuals in periods of pre-puberty and puberty may have different development rates [6]. The physical growth of children at an early maturation level is greater than of children at a delayed maturation level, and this situation can provide advantage in physical activities. The maturation level can be effective on physical activity performance [7]. The bone age and puberty phase are mentioned as biological maturation indicators in athletic performance studies [8]. The main requirements of physical activity are basic performance characteristics, such as strength, speed, endurance, power, agility. These performance parameters are affected by body composition. Therefore, the maturation level is a important factor for body composition and physical performance. The muscle mass of body is a important factor at strength and power sports, and muscle mass increase is triggered by maturation. Also, the determination of biological maturation role on musculoskeletal development is important in terms of healthy development of adolescent individuals [9]. Deprez et al. [10] indicated that early maturation could increase the selection possibility during identification of young soccer players. Many sport disciplines requires spesific physical performance parameters at different levels. The talent selection process involves selection of children at an optimal physical fitness level in different sports disciplines. The determination of maturation level of children can assist sports sciences professionals in the talent selection process. The determination of performance parameters differences among early, normal and delayed maturation levels is also important in many sport disciplines for the effective talent selection process. In this context, the aim of the study was to examine some physical fitness parameters of students aged eight years according to their maturation level and gender.

\section{MATERIAL AND METHODS}

\section{PARTICIPANTS}

The research group consisted of 535 primary education students (age: 8.19 \pm 2.25 years, weight: $31.34 \pm 11.84 \mathrm{~kg}$, height: $131.02 \pm 13.66 \mathrm{~cm}), 300$ of them being boys (age: $8.25 \pm 2.32$ years, weight: $32.41 \pm 12.82 \mathrm{~kg}$, height: $131.69 \pm 3.59 \mathrm{~cm}$ ) and 235 girls (age: $8.12 \pm 2.16$ years, weight: $29.98 \pm 10.33$ $\mathrm{kg}$, height: $130.16 \pm 13.31 \mathrm{~cm}$ ). All students and their parents were informed about measurement tests. The informed voluntary consent form was filled by students' parents, and all students participated in the study voluntarily. The study was performed in consistence with the Helsinki Declaration. 


\section{EXPERIMENTAL DESIGN}

Anthropometric Measurements

The weight, height, sitting height, stroke length, stroke rate, leg length and body mass index parameters as anthropometric measurement parameters were measured. The height values of students were measured with a stadiometer (Holtain Ltd., Crymych, Dyfed, UK). The weight values were measured with $0.1 \mathrm{~kg}$ precision with an electronic weight scale calibrated manually (SECA 709, Hamburg, Germany). The sitting height values of students were measured with a Harpenden sitting height device (Holtain Ltd., Crymych, Dyfed, UK). The hips, backs and occiputs of students were placed to device on the vertical plane [11]. The position of heads was adjusted according to the Frankfurt plane of anatomy [11]. The sitting height was recorded with $0.1 \mathrm{~cm}$ precision. The stroke length was measured by a sliding caliper with the arms opened to the side on the horizontal axis and leaning against the wall in a standing position (Holtain Ltd., Crymych, Dyfed, UK). The distance between the middle fingers of both hands was measured as the stroke length with $0.01 \mathrm{~cm}$ precision. The stroke rate value was determined as difference between stroke length and height values. The length between tibial condyle and medial malleolus was measured as leg length [12-14] with $0.1 \mathrm{~cm}$ precision used a sliding caliper (Holtain Ltd., Crymych, Dyfed, UK). The body mass index was determined by dividing the body weight value expressed in $\mathrm{kg}$ by the square of height value expressed in $\mathrm{m}[15,16]$.

\section{Flexibility Test}

The flexibility values of students were measured with a sit-and-reach test. The sit-and-reach test was carried out using a sit-and reach-apparatus (Lafayette 01285A, Lafayette Instruments, USA). A scale on it indicates the reach distance of students during the sit-and-reach test. The calibration of test bench was done manually before the test. The students performed the test barefoot. The students sat on the floor and leaned soles of their feet against to test bench. Then students pushed the apparatus of the sit-and-reach bench with their hands, and the reach distance was recorded with $0.1 \mathrm{~cm}$ precision.

\section{Handgrip Strength Test}

The handgrip strength of students was determined with a handgrip dynamometer (Takei Scientific Instruments, Tokyo, Japan). The right and left handgrip strength was tested. The handgrip strength measurement was carried out at 450 of shoulder flexion. The students gripped the dynamometer with the maximum effort for three seconds. The test was carried out as two repetitions for both hands. The highest value of three repetitions was recorded in $\mathrm{kg}$ as the test score.

\section{Vertical Jump Tests}

Students' vertical jump ability was determined with squat jump and countermovement jump tests. The tests were carried out on different days. The Optojump measurement system consisting of two bars attached to a computer was used for vertical jump tests (Optojump, Microgate, Bolzano, Italy). The students did vertical jumps between two bars of measurement system. The two bars of the measurement system were connected to computer and the vertical jump values were calculated by system software. The squat jump test was used to determine explosive strength of leg muscles. The test was carried out at $90^{\circ}$ knee flexion with both hands at the waist. The students tried to jump with maximum effort. The countermovement jump was a test measuring 
elastic strength of leg muscles as well as the explosive strength. The students stood in the position of full knee extension with both hands at the waist. After taking position at $90^{\circ}$ knee flexion, students carried out vertical jump with maximum effort. Both of the vertical jump tests were carried out as three repetitions, and the best value was recorded in $\mathrm{cm}$ as the vertical jump value.

\section{Tapping (Skipping) Test}

The tapping (skipping) test was carried out to determine the step frequency during a running exercise. During the tapping (skipping) test, the step frequency, flight times and contact times to the ground by the right and left foot, step/speed ratio parameters were measured by Optojump measurement system (Optojump, Microgate, Bolzano, Italy). The Optojump measurement system has two bars. One of these bars has a transmitter system and other one has a receiver system. The bars of system are paralelly set and the system measures flight times, contact times, step frequency and step/speed ratio parameters in $1 \mathrm{~ms}$ precision with these bars. The receiver bar of the system includes electronic diodes emitting light. When the jump exercise is carried out between the transmitter and receiver bars, the receiver bar interrupts light and the system's timer measures the contact time as the total time of contact to ground and the flight time as the total time among contacts to ground. The tapping (skipping) test lasted 15 seconds, and students tried to step with maximum effort during 15 seconds test duration [17]. The test parameters were determined by software of the Optojump system connected to a computer.

\section{Postural Sway Test}

The postural sway test was carried out to evaluate mobility and the stationary position at the center of gravity over a support leg. The postural sway test was carried out on a portable force plate (Kistler 9807, Kistler Instrumente AG, Winterthur, Switzerland). The force plate measures total, anterior-posterior (throughout Y axis) and medial-lateral (throughout $\mathrm{X}$ axis) postural sway values in millimeters ( $\mathrm{mm}$ ) with $50 \mathrm{~Hz}$ frequency. The students stood on a force plate in a double leg stance position with opened eyes without losing balance for 10 seconds [18]. The anteriorposterior and medial lateral postural sway values were automatically determined with the device software on a computer connected to the plate. It is stated that lower postural sway values indicate a higher balance ability.

\section{Visual Reaction Test}

Students' visual reaction time was determined by an electronic reaction measurement system (Witty Sem, Microgate, Bolzano, Italy). The system consisted of six visual reaction lamps which include blue, green and red light stimulants. The visual reaction lamps were attached to tripods and placed in the half circle with 1.8 meter radius of the basketball pitch free throw line on the parquet floor of an indoor sports hall. When the test began, students tried to touch lamps giving blue light alerts randomly. The test was finished when sixty light alerts were completed. The mean visual reaction time values during test were determined by software of reaction measurement system with $0.01 \mathrm{sec}$. precision.

\section{Reaction Test}

The reaction time was determined by an electronic reaction time measurement system (Smart Speed, Fusion Sport, Brisbane, Australia). The system consisted of a light stimulant, a photocell gate, a reaction mat and a computer. The $5 \mathrm{~m}$ sprint run was carried out for the reaction test. The photocell gate of the system was set at the finish line of $5 \mathrm{~m}$ sprint run. The students carried out warming exercises before test. The reaction mat of system was set at the start 
line of test. The students stepped to the reaction mat with the dominant foot and waited for the light stimulant positioned in finish photocell at start position. The light stimulant of system was given and the students sprinted to the finish photocell. The students completed the $5 \mathrm{~m}$ distance with maximum effort. The test was carried out as three repetitions and the best reaction time value was accepted as the test score. The students' reaction time was calculated with an accuracy of 0.01 seconds as the difference between the time of emitting the light stimulus and the time of leaving the mat.

\section{Sprint Test}

The $20 \mathrm{~m}$ sprint time was carried out to determine students' sprint ability. The $20 \mathrm{~m}$ test track was set on a parquet floor of an indoor sport hall. The wireless photocell system was used to determine the sprint times (Witty, Microgate, Bolzano, Italy). The photocells were placed at the start and the finish line of $20 \mathrm{~m}$ test track. The warming exercises were carried out before the test. The time started automatically when the start photocell was passed. The 20 meters test time was determined automatically with 0.01 seconds precision when the student passed the 20 meters photocell and stopped the time. The students' test time was recorded by an electronic tracking device of a wireless photocell. The test was carried out in three repetitions with rest intervals, and the best sprint time was recorded as the 20 meters sprint time.

\section{Agility Test}

The Pro-agility test was carried out to determine students' ability to change direction quickly. The test times were determined by a wireless electronic photocell system (Witty, Microgate, Bolzano, Italy). The test track is demonstrated in Figure 1 [19]. The Pro-agility test consists of total 9.14 meters distances as 4.57 meters to the right and the left of the start line. The gate of a wireless photocell was put in the start line of the test. After warming exercises, the students carried out the test. The students started test time when passing the start line. The test consists of runs to 4.57 meters to the right, 9.14 meters to the left and back to the start line, respectively (Figure 1). The test time was automatically recorded by an electronic photocell system. The students carried out the Pro-agility test in three repetitions with rest intervals, and the shortest test time was determined as the agility test value.

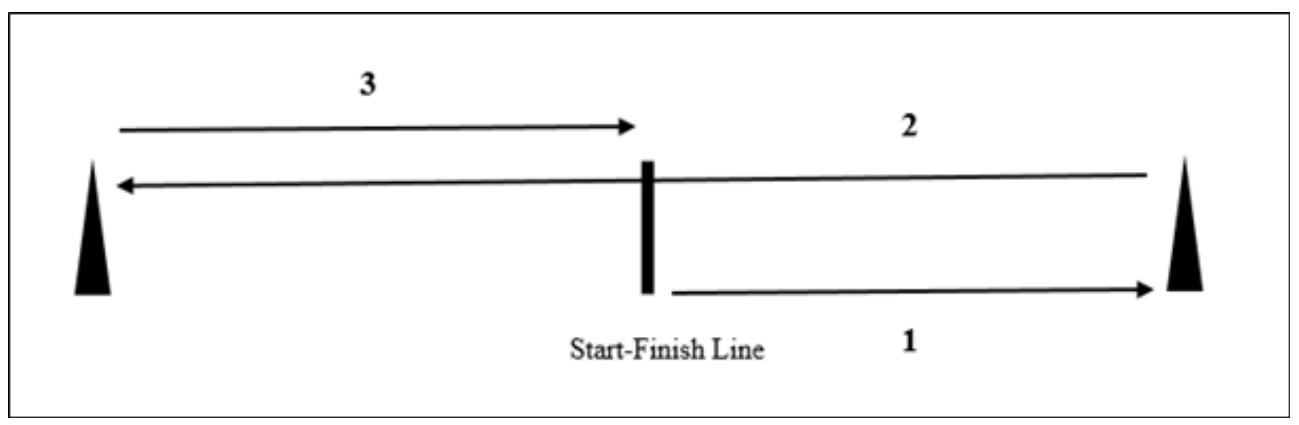

Fig. 1. Pro-Agility Test [19]

\section{Determination of the level of maturation}

The maturation offset of students was calculated by the maturation offset formula of Malina et al. [20], separately for boy and girl students. The maturation offset formula of Malina et al. [20] is given below: 
Boys: maturity offset $=-9.236+[0.0002708 \times$ (leg length $\mathrm{x}$ sitting height) $]+$ $[-0.001663 \mathrm{x}$ (age $\mathrm{x}$ leg length) $]+[0.007216 \mathrm{x}$ (age $\mathrm{x}$ sitting height) $]+$ [0.02292 x (mass / height ratio $\mathrm{x} 100)]$.

Girls: maturity offset $=-9.376+0.0001882$ (leg length $\mathrm{x}$ sitting height) + 0.0022 (age $\mathrm{x}$ leg length) +0.005841 (age $\mathrm{x}$ sitting height) -0.002658 (age $\mathrm{x}$ weight) +0.07693 (mass /height ratio $\mathrm{x} 100$ )].

A high positive correlation between skeletal age and maturation offsets was reported [21]. The maturation offset values were standardized with z-score (z-score = raw maturation offset - mean of raw maturation offsets / standart deviation of raw maturation offsets). The categorization of maturation levels was determined by z-score values of maturation offsets [20], and it is presented in Table 1.

Table 1. Maturation level categorization according to z-scores of maturation offsets [20]

\begin{tabular}{cc} 
z-Score of Maturation Offset & Maturation Level \\
\hline Over +1.0 & Early \\
-1.0 to +1.0 & Normal \\
Below -1.0 & Delayed \\
\hline
\end{tabular}

\section{STATISTICAL ANALYSIS}

The study data was analyzed in SPSS 22.0 statistic package program (SPSS Inc., IBM, Chicago, USA). The data is presented as descriptive statistics (mean \pm standard deviation). The normality of data distribution was tested by the Kolmogorov-Smirnov test. The test parameters were compared according to students' gender and maturation level. The parametric study data were compared with independent samples T-test according to gender and with one way ANOVA test according to the maturation level. The variance homogeneity in independent samples of the T-test and one-way ANOVA analysis was determined with Levene's test. The comparison of non-parametric data was done with the Mann Whitney-U test, according to gender, and with the Kruskal Wallis-H test, according to the maturation level. The pairwise comparisons of significant differences between groups in one-way ANOVA test were analyzed with Tukey HSD test and Dunnett C test according to variances homogeneity. The Mann Whitney-U test with Bonferroni correction was used for pairwise comparisons of significant differences between groups in the Kruskal Wallis- $\mathrm{H}$ test. The significance level of statistical analysis was set as $\mathrm{p}<0.05$.

\section{RESULTS}

The Table 2 is depicting a descriptive statistics of test parameters of boy and girl students.

According to Table 3, there was a significant difference between boy and girl students in terms of sitting height, stroke rate, flexibility, squat jump, reaction, jump and right foot flight time parameters $(\mathrm{p}<0.05)$. Also, a better flexibility value of girl students was noted in comparison to boys $(p<0.05)$. 
Table 2. Descriptive statistics of test parameters of boy and girl students

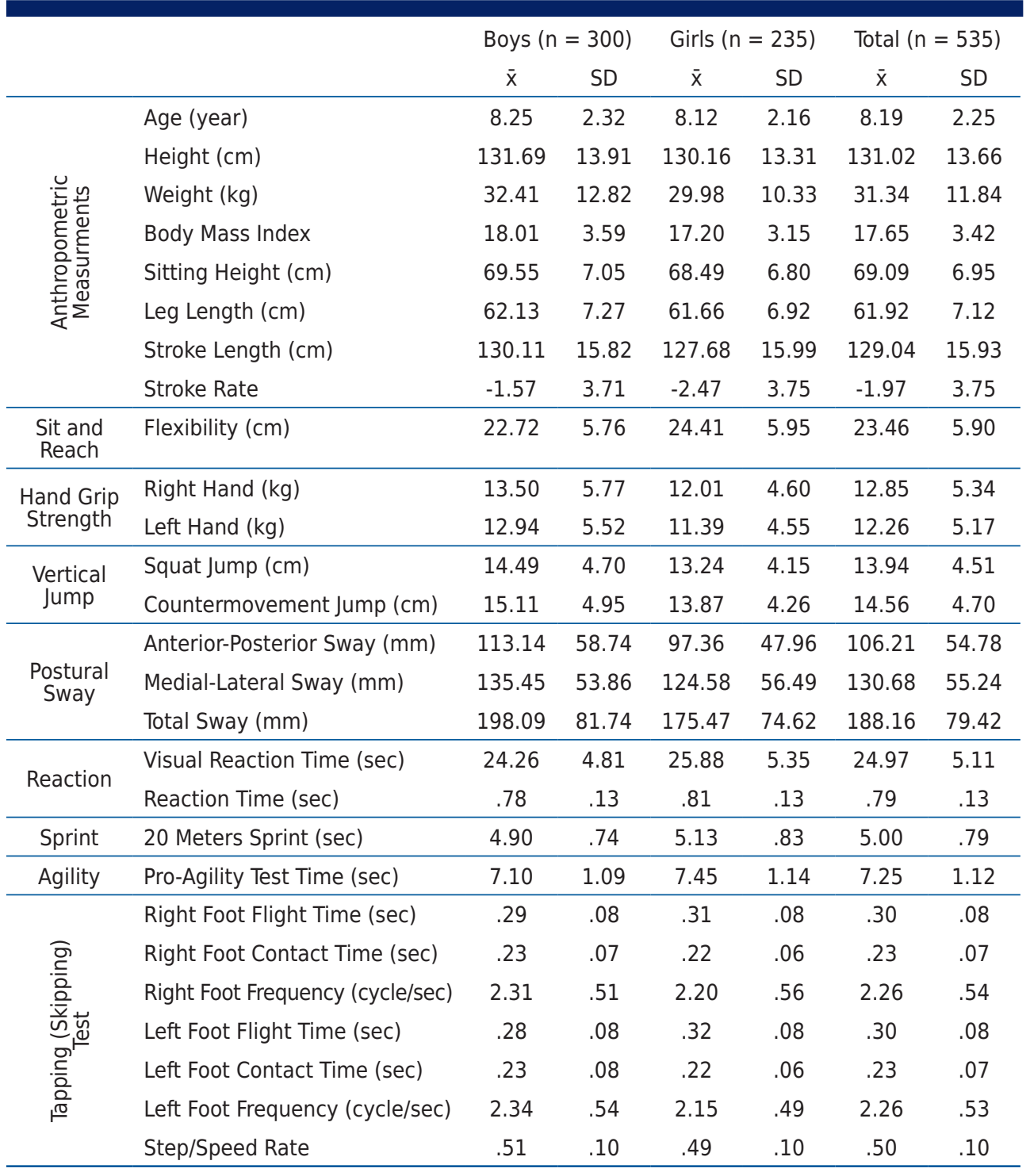

Table 3. Independent samples t-test analysis table of the parametric test values according to gender

\begin{tabular}{|c|c|c|c|c|c|c|}
\hline & Gender & $n$ & $\bar{x}$ & SD & $\mathrm{t}$ & $\mathrm{p}$ \\
\hline \multirow{2}{*}{ Sitting Height (cm) } & Boys & 300 & 69.55 & 7.05 & \multirow{2}{*}{1.752} & \multirow{2}{*}{.080} \\
\hline & Girls & 235 & 68.49 & 6.80 & & \\
\hline \multirow{2}{*}{ Stroke rate } & Boys & 300 & -1.57 & 3.71 & \multirow{2}{*}{2.759} & \multirow{2}{*}{$.006 *$} \\
\hline & Girls & 235 & -2.47 & 3.75 & & \\
\hline \multirow{2}{*}{ Flexibility (cm) } & Boys & 300 & 22.72 & 5.76 & \multirow{2}{*}{-3.320} & \multirow{2}{*}{$.001 *$} \\
\hline & Girls & 235 & 24.41 & 5.95 & & \\
\hline \multirow{2}{*}{ Squat Jump (cm) } & Boys & 300 & 14.49 & 4.70 & \multirow{2}{*}{3.224} & \multirow{2}{*}{$.001 *$} \\
\hline & Girls & 235 & 13.24 & 4.15 & & \\
\hline \multirow{2}{*}{ Reaction Time (sec) } & Boys & 300 & .78 & .13 & \multirow{2}{*}{-3.234} & \multirow{2}{*}{$.001 *$} \\
\hline & Girls & 235 & .81 & .13 & & \\
\hline \multirow{2}{*}{ Right Foot Flight Time (sec) } & Boys & 300 & .29 & .08 & \multirow{2}{*}{-2.746} & \multirow{2}{*}{$.006 *$} \\
\hline & Girls & 235 & ,31 &, 08 & & \\
\hline
\end{tabular}

$* \mathrm{p}<0.05$ 
According to Table 4, a significant difference was determined between boy and girl students with regard to all non-parametric test values without leg length, stroke length, left foot contact time and step/speed ratio parameters $(\mathrm{p}<0.05)$. As regards the postural sway values, girl students' balance ability was better than boys'.

Table 4. The Mann Whitney-U test analysis table of the non-parametric test values according to gender

\begin{tabular}{|c|c|c|c|c|c|c|c|}
\hline & Gender & $\mathrm{n}$ & $\bar{x}$ & SD & Median & U & $p$ \\
\hline \multirow{2}{*}{ Body Mass Index } & Boys & 300 & 18.01 & 3.59 & 16.88 & \multirow{2}{*}{30506.00} & \multirow{2}{*}{$.008 *$} \\
\hline & Girls & 235 & 17.20 & 3.15 & 16.34 & & \\
\hline \multirow{2}{*}{ Leg Length (cm) } & Boys & 300 & 62.13 & 7.27 & 62.05 & \multirow{2}{*}{33960.00} & \multirow{2}{*}{.467} \\
\hline & Girls & 235 & 61.66 & 6.92 & 61.00 & & \\
\hline \multirow{2}{*}{ Stroke Length (cm) } & Boys & 300 & 130.11 & 15.82 & 130.45 & \multirow{2}{*}{31766.50} & \multirow{2}{*}{.050} \\
\hline & Girls & 235 & 127.68 & 15.99 & 126.00 & & \\
\hline \multirow{2}{*}{$\begin{array}{l}\text { Right Hand Strength } \\
(\mathrm{kg})\end{array}$} & Boys & 300 & 13.50 & 5.77 & 12.75 & \multirow{2}{*}{29079.50} & \multirow{2}{*}{$.001 *$} \\
\hline & Girls & 235 & 12.01 & 4.60 & 11.40 & & \\
\hline \multirow{2}{*}{$\begin{array}{l}\text { Left Hand Strength } \\
(\mathrm{kg})\end{array}$} & Boys & 300 & 12.94 & 5.52 & 12.05 & \multirow{2}{*}{28371.50} & \multirow{2}{*}{$.000 *$} \\
\hline & Girls & 235 & 11.39 & 4.55 & 10.70 & & \\
\hline \multirow{2}{*}{$\begin{array}{l}\text { Countermovement } \\
\text { Jump }(\mathrm{cm})\end{array}$} & Boys & 300 & 15.11 & 4.95 & 14.80 & \multirow{2}{*}{29773.50} & \multirow{2}{*}{$.003 *$} \\
\hline & Girls & 235 & 13.87 & 4.26 & 13.30 & & \\
\hline \multirow{2}{*}{$\begin{array}{l}\text { Anterior-Posterior Sway } \\
(\mathrm{mm})\end{array}$} & Boys & 300 & 113.14 & 58.74 & 98.26 & \multirow{2}{*}{28713.00} & \multirow{2}{*}{$.000 *$} \\
\hline & Girls & 235 & 97.36 & 47.96 & 89.09 & & \\
\hline \multirow{2}{*}{$\begin{array}{l}\text { Medial-Lateral Sway } \\
(\mathrm{mm})\end{array}$} & Boys & 300 & 135.45 & 53.86 & 121.90 & \multirow{2}{*}{29791.00} & \multirow{2}{*}{$.002 *$} \\
\hline & Girls & 235 & 124.58 & 56.49 & 113.90 & & \\
\hline \multirow{2}{*}{ Total Sway (mm) } & Boys & 300 & 198.09 & 81.74 & 179.85 & \multirow{2}{*}{28671.50} & \multirow{2}{*}{$.000 *$} \\
\hline & Girls & 235 & 175.47 & 74.62 & 161.60 & & \\
\hline Vicual Roartion (cos) & Boys & 300 & 24.26 & 4.81 & 23.55 & 2821050 & $0 \cap 0 *$ \\
\hline 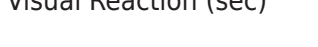 & Girls & 235 & 25.88 & 5.35 & 24.61 & 20310.30 & .000 \\
\hline 20 Meterc Snrint (cer) & Boys & 300 & 4.90 & .74 & 4.82 & 3032000 & ก06* \\
\hline 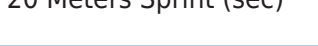 & Girls & 235 & 5.13 & .83 & 4.92 & (2) & .000 \\
\hline Pro-Agility Test Time & Boys & 300 & 7.10 & 1.09 & 6.82 & 2779950 & $000 *$ \\
\hline (sec) & Girls & 235 & 7.45 & 1.14 & 7.20 & 21199.50 & $.000^{4}$ \\
\hline Right Foot Contact Time & Boys & 300 & .23 & .07 & .21 & 3138400 & $020 *$ \\
\hline (sec) & Girls & 235 & .22 & .06 & .21 & 31384.00 & $.0 \angle 9^{n}$ \\
\hline Right Foot Frequency & Boys & 300 & 2.31 & .51 & 2.26 & 2979200 & $002 *$ \\
\hline (cycle/sec) & Girls & 235 & 2.20 & .56 & 2.11 & 29192.00 & .002 \\
\hline Left Foot Flight Time & Boys & 300 & .28 & .08 & .27 & 2536800 & $000 *$ \\
\hline (sec) & Girls & 235 & .32 & .08 & .31 & 25000.00 & \\
\hline Left Foot Contact Time & Boys & 300 & .23 & .08 & .22 & 3227150 & 003 \\
\hline (sec) & Girls & 235 & .22 & .06 & .21 & 3221.00 & כדכט. \\
\hline Left Foot Frequency & Boys & 300 & 2.34 & .54 & 2.33 & 2668150 & $000 *$ \\
\hline (cycle/sec) & Girls & 235 & 2.15 & .49 & 2.09 & 20001.50 & .000 \\
\hline Sten/Sneed Ratin & Boys & 300 & .51 & .10 & .50 & 3268250 & 148 \\
\hline step/speeu natio & Girls & 235 & .49 & .10 & .50 & 52002.50 & .140 \\
\hline
\end{tabular}

$* \mathrm{p}<0.05$ 
Table 5. Descriptive statistics of test parameters of boy students according to their maturation level

\begin{tabular}{|c|c|c|c|c|c|c|c|}
\hline & & \multicolumn{2}{|c|}{ Early $(n=49)$} & \multicolumn{2}{|c|}{ Normal $(n=209)$} & \multicolumn{2}{|c|}{ Delayed $(n=42)$} \\
\hline & & $\bar{x}$ & SD & $\bar{x}$ & SD & $\bar{x}$ & SD \\
\hline \multirow{8}{*}{ 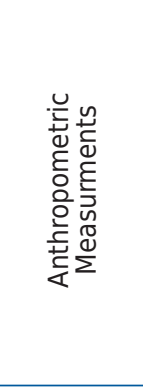 } & Age (year) & 8.40 & 2.41 & 8.30 & 2.28 & 7.78 & 2.45 \\
\hline & Height (cm) & 141.03 & 14.77 & 131.33 & 12.57 & 122.60 & 12.88 \\
\hline & Weight (kg) & 43.40 & 16.70 & 31.50 & 11.01 & 24.12 & 6.13 \\
\hline & Body Mass Index & 21.03 & 4.20 & 17.73 & 3.26 & 15.83 & 1.87 \\
\hline & Sitting Height (cm) & 74.74 & 6.86 & 69.41 & 6.38 & 64.24 & 6.27 \\
\hline & Leg Length (cm) & 66.28 & 8.37 & 61.92 & 6.56 & 58.36 & 7.08 \\
\hline & Stroke Length $(\mathrm{cm})$ & 139.44 & 16.62 & 129.85 & 14.69 & 120.53 & 14.45 \\
\hline & Stroke Rate & -1.58 & 4.75 & -1.47 & 3.60 & -2.07 & 2.78 \\
\hline $\begin{array}{l}\text { Sit and } \\
\text { Reach }\end{array}$ & Flexibility (cm) & 24.69 & 5.76 & 22.45 & 5.85 & 21.75 & 4.81 \\
\hline \multirow{2}{*}{$\begin{array}{l}\text { Hand Grip } \\
\text { Strength }\end{array}$} & Right Hand (kg) & 15.99 & 5.77 & 13.54 & 5.79 & 10.41 & 4.13 \\
\hline & Left Hand (kg) & 15.53 & 5.51 & 12.92 & 5.53 & 10.06 & 3.84 \\
\hline \multirow{2}{*}{$\begin{array}{l}\text { Vertical } \\
\text { Jump }\end{array}$} & Squat Jump (cm) & 13.68 & 3.60 & 14.90 & 4.86 & 13.43 & 4.85 \\
\hline & Countermovement Jump (cm) & 14.58 & 3.68 & 15.41 & 5.13 & 14.25 & 5.25 \\
\hline \multirow{3}{*}{$\begin{array}{l}\text { Postural } \\
\text { Sway }\end{array}$} & Anterior-Posterior Sway (mm) & 91.84 & 29.39 & 115.72 & 61.86 & 125.15 & 63.38 \\
\hline & Medial-Lateral Sway (mm) & 120.54 & 39.87 & 137.11 & 58.22 & 144.60 & 41.62 \\
\hline & Total Sway (mm) & 171.78 & 52.42 & 201.03 & 87.47 & 214.21 & 74.54 \\
\hline \multirow{2}{*}{ Reaction } & Visual Reaction Time (sec) & 23.64 & 3.86 & 24.15 & 4.81 & 25.52 & 5.60 \\
\hline & Reaction Time (sec) & .80 & .12 & .78 & .13 & .73 & .12 \\
\hline Sprint & 20 Meters Sprint (sec) & 4.92 & .72 & 4.84 & .68 & 5.21 & .93 \\
\hline Agility & Pro-Agility Test Time (sec) & 7.16 & 1.10 & 7.03 & 1.04 & 7.37 & 1.27 \\
\hline \multirow{7}{*}{ 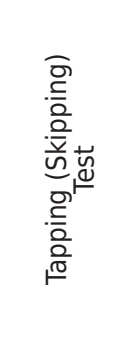 } & Right Foot Flight Time (sec) & .30 & .09 & .29 & .08 & .30 & .10 \\
\hline & Right Foot Contact Time (sec) & .25 & .08 & .23 & .07 & .23 & .08 \\
\hline & Right Foot Frequency (cycle/sec) & 2.28 & .66 & 2.30 & .46 & 2.38 & .57 \\
\hline & Left Foot Flight Time (sec) & .29 & .09 & .28 & .07 & .30 & .10 \\
\hline & Left Foot Contact Time (sec) & .24 & .09 & .23 & .07 & .23 & .08 \\
\hline & Left Foot Frequency (cycle/sec) & 2.26 & .65 & 2.36 & .51 & 2.33 & .55 \\
\hline & Step/Speed Rate & .50 & .12 & .51 & .09 & .49 & .09 \\
\hline
\end{tabular}

The sitting height, leg and stroke length, flexibility, right and left hand strength, total postural sway parameters were significantly differentiated among boy students at an early, normal and delayed maturation level, according to analysis results $(\mathrm{p}<0.05)$. Boy students at an early maturation level had higher test values than boy students at other maturation levels, except for the total postural sway parameter, when the pairwise comparison results between the groups were examined. It was determined that boy students at an early maturation level had lower total postural sway values than boy students at other maturation levels. This finding indicated that the balance ability of boy students at early maturation level was better than the balance ability of boy students at a normal and delayed maturation level. 
Table 6. One-way ANOVA analysis table of the parametric test values of boy students according to their maturation level

\begin{tabular}{|c|c|c|c|c|c|c|c|}
\hline & $\begin{array}{l}\text { Maturation } \\
\text { Level }\end{array}$ & $\mathrm{n}$ & $\bar{x}$ & SD & $\mathrm{F}$ & $\mathrm{p}$ & $\begin{array}{l}\text { Significant } \\
\text { Difference }\end{array}$ \\
\hline \multirow{3}{*}{ Sitting Height (cm) } & Early & 49 & 74.74 & 6.86 & \multirow{3}{*}{30.197} & \multirow{3}{*}{$.000 *$} & \multirow{3}{*}{$\begin{array}{l}\text { Early-Normal } \\
\text { Early-Delayed } \\
\text { Normal-Delayed }\end{array}$} \\
\hline & Normal & 209 & 69.41 & 6.38 & & & \\
\hline & Delayed & 42 & 64.24 & 6.27 & & & \\
\hline \multirow{3}{*}{ Leg Length (cm) } & Early & 49 & 66.28 & 8.37 & \multirow{3}{*}{14.954} & \multirow{3}{*}{$.000 *$} & \multirow{3}{*}{$\begin{array}{l}\text { Early-Normal } \\
\text { Early-Delayed } \\
\text { Normal-Delayed }\end{array}$} \\
\hline & Normal & 209 & 61.92 & 6.56 & & & \\
\hline & Delayed & 42 & 58.36 & 7.08 & & & \\
\hline \multirow{3}{*}{ Stroke Length $(\mathrm{cm})$} & Early & 49 & 139.44 & 16.62 & \multirow{3}{*}{18.094} & \multirow{3}{*}{$.000 *$} & \multirow{3}{*}{$\begin{array}{l}\text { Early-Normal } \\
\text { Early-Delayed } \\
\text { Nomal-Delayed }\end{array}$} \\
\hline & Normal & 209 & 129.85 & 14.69 & & & \\
\hline & Delayed & 42 & 120.53 & 14.45 & & & \\
\hline \multirow{3}{*}{ Stroke Rate } & Early & 49 & -1.58 & 4.75 & \multirow{3}{*}{.445} & \multirow{3}{*}{.641} & \\
\hline & Normal & 209 & -1.47 & 3.60 & & & \\
\hline & Delayed & 42 & -2.07 & 2.78 & & & \\
\hline \multirow{3}{*}{ Flexibility (cm) } & Early & 49 & 24.69 & 5.76 & \multirow{3}{*}{3.761} & \multirow{3}{*}{$.024 *$} & \multirow{3}{*}{$\begin{array}{l}\text { Early-Normal } \\
\text { Early-Delayed }\end{array}$} \\
\hline & Normal & 209 & 22.45 & 5.85 & & & \\
\hline & Delayed & 42 & 21.75 & 4.81 & & & \\
\hline \multirow{3}{*}{$\begin{array}{l}\text { Right Hand Strength } \\
(\mathrm{kg})\end{array}$} & Early & 49 & 15.99 & 5.77 & \multirow{3}{*}{11.027} & \multirow{3}{*}{$.000 *$} & \multirow{3}{*}{$\begin{array}{l}\text { Early-Normal } \\
\text { Early-Delayed } \\
\text { Normal-Delayed }\end{array}$} \\
\hline & Normal & 209 & 13.54 & 5.79 & & & \\
\hline & Delayed & 42 & 10.41 & 4.13 & & & \\
\hline \multirow{3}{*}{$\begin{array}{l}\text { Left Hand Strength } \\
(\mathrm{kg})\end{array}$} & Early & 49 & 15.53 & 5.51 & \multirow{3}{*}{11.663} & & Farly-Normal \\
\hline & Normal & 209 & 12.92 & 5.53 & & $.000^{*}$ & Early-Delayed \\
\hline & Delayed & 42 & 10.06 & 3.84 & & & \\
\hline & Early & 49 & 13.68 & 3.60 & & & \\
\hline Squat Jump (cm) & Normal & 209 & 14.90 & 4.86 & 2.607 & .075 & \\
\hline & Delayed & 42 & 13.43 & 4.85 & & & \\
\hline & Early & 49 & 14.58 & 3.68 & & & \\
\hline $\begin{array}{l}\text { Countermovement } \\
\text { Jump (cm) }\end{array}$ & Normal & 209 & 15.41 & 5.13 & 1.289 & .277 & \\
\hline & Delayed & 42 & 14.25 & 5.25 & & & \\
\hline & Early & 49 & 171.78 & 52.42 & & & \\
\hline Total Sway (mm) & Normal & 209 & 201.03 & 87.47 & 3.549 & $.030 *$ & Early-Delayed \\
\hline & Delayed & 42 & 214.21 & 74.54 & & & \\
\hline & Early & 49 & .80 & .12 & & & \\
\hline Reaction Time (sec) & Normal & 209 & .78 & .13 & 2.898 & .057 & \\
\hline & Delayed & 42 & .73 & .12 & & & \\
\hline & Early & 49 & 2.28 & .66 & & & \\
\hline $\begin{array}{l}\text { Right Foot Frequency } \\
\text { (cycle/sec) }\end{array}$ & Normal & 209 & 2.30 & .46 & .508 & .602 & \\
\hline & Delayed & 42 & 2.38 & .57 & & & \\
\hline & Early & 49 & 2.26 & .65 & & & \\
\hline $\begin{array}{l}\text { Left Foot Frequency } \\
\text { (cycle/sec) }\end{array}$ & Normal & 209 & 2.36 & .51 & .777 & .461 & \\
\hline & Delayed & 42 & 2.33 & .55 & & & \\
\hline
\end{tabular}

$* \mathrm{p}<0.05$

The analysis results of non-parametric test parameters indicated that the anterior-posterior and medial-lateral sway value of boy students at an early maturation level was the lowest among the three groups. Accordingly, the balance ability with regard to the anterior-posterior sway parameter of boy students at early maturation level was better than in the other groups. On the other hand, a significant difference was found between early and delayed maturation groups 
in terms of medial-lateral sway value $(\mathrm{p}<0.05)$. This finding indicated that the balance ability in terms of the medial-lateral sway parameter was at a better level in the early maturation group as compared to the delayed maturation group.

Table 7. The Kruskal Wallis-H analysis table of the non-parametric test values of boy students according to their maturation level

\begin{tabular}{|c|c|c|c|c|c|c|c|c|}
\hline & $\begin{array}{l}\text { Maturation } \\
\text { Level }\end{array}$ & $\mathrm{n}$ & $\bar{x}$ & SD & Median & $\begin{array}{l}\text { Chi- } \\
\text { square }\end{array}$ & $\mathrm{p}$ & $\begin{array}{l}\text { Significant } \\
\text { Difference }\end{array}$ \\
\hline \multirow{3}{*}{ Body Mass Index } & Early & 49 & 21.03 & 4.20 & 20.24 & \multirow{3}{*}{46.516} & \multirow{3}{*}{$.000 *$} & \multirow{3}{*}{$\begin{array}{l}\text { Early-Normal } \\
\text { Early-Delayed } \\
\text { Nomal-Delayed }\end{array}$} \\
\hline & Normal & 209 & 17.73 & 3.26 & 16.82 & & & \\
\hline & Delayed & 42 & 15.83 & 1.87 & 15.76 & & & \\
\hline \multirow{3}{*}{$\begin{array}{l}\text { Anterior- } \\
\text { Posterior Sway } \\
(\mathrm{mm})\end{array}$} & Early & 49 & 91.84 & 29.39 & 85.59 & \multirow{3}{*}{10.141} & \multirow{3}{*}{$.006 *$} & \multirow{3}{*}{$\begin{array}{l}\text { Early-Normal } \\
\text { Early-Delayed }\end{array}$} \\
\hline & Normal & 209 & 115.72 & 61.86 & 99.75 & & & \\
\hline & Delayed & 42 & 125.15 & 63.38 & 117.56 & & & \\
\hline \multirow{3}{*}{$\begin{array}{l}\text { Medial-Lateral } \\
\text { Sway (mm) }\end{array}$} & Early & 49 & 120.54 & 39.87 & 116.41 & \multirow{3}{*}{8.321} & \multirow{3}{*}{$.016^{*}$} & \multirow{3}{*}{ Early-Delayed } \\
\hline & Normal & 209 & 137.11 & 58.22 & 119.90 & & & \\
\hline & Delayed & 42 & 144.60 & 41.62 & 141.65 & & & \\
\hline \multirow{3}{*}{$\begin{array}{l}\text { Visual Reaction } \\
\text { Time (sec) }\end{array}$} & Early & 49 & 23.64 & 3.86 & 22.80 & \multirow{3}{*}{2.029} & \multirow{3}{*}{.363} & \\
\hline & Normal & 209 & 24.15 & 4.81 & 23.57 & & & \\
\hline & Delayed & 42 & 25.52 & 5.60 & 23.90 & & & \\
\hline \multirow{3}{*}{$\begin{array}{l}20 \text { Meters Sprint } \\
\text { (sec) }\end{array}$} & Early & 49 & 4.92 & .72 & 4.82 & \multirow{3}{*}{4.588} & \multirow{3}{*}{.101} & \\
\hline & Normal & 209 & 4.84 & .68 & 4.82 & & & \\
\hline & Delayed & 42 & 5.21 & .93 & 5.20 & & & \\
\hline \multirow{3}{*}{$\begin{array}{l}\text { Pro-Agility Test } \\
\text { Time (sec) }\end{array}$} & Early & 49 & 7.16 & 1.10 & 6.87 & \multirow{3}{*}{2.145} & \multirow{3}{*}{.342} & \\
\hline & Normal & 209 & 7.03 & 1.04 & 6.81 & & & \\
\hline & Delayed & 42 & 7.37 & 1.27 & 7.11 & & & \\
\hline \multirow{3}{*}{$\begin{array}{l}\text { Right Foot Flight } \\
\text { Time (sec) }\end{array}$} & Early & 49 & .30 & .09 & .29 & \multirow{3}{*}{.587} & \multirow{3}{*}{.745} & \\
\hline & Normal & 209 & .29 & .08 & .28 & & & \\
\hline & Delayed & 42 & .30 & .10 & .28 & & & \\
\hline \multirow{3}{*}{$\begin{array}{l}\text { Right Foot } \\
\text { Contact Time } \\
\text { (sec) }\end{array}$} & Early & 49 & .25 & .08 & .22 & \multirow{3}{*}{1.196} & \multirow{3}{*}{.550} & \\
\hline & Normal & 209 & .23 & .07 & .21 & & & \\
\hline & Delayed & 42 & .23 & .08 & .21 & & & \\
\hline \multirow{3}{*}{$\begin{array}{l}\text { Left Foot Flight } \\
\text { Time (sec) }\end{array}$} & Early & 49 & .29 & .09 & .28 & & & \\
\hline & Normal & 209 & .28 & .07 & .27 & 2.268 & .322 & \\
\hline & Delayed & 42 & .30 & .10 & .28 & & & \\
\hline & Early & 49 & .24 & .09 & .22 & & & \\
\hline $\begin{array}{l}\text { Left Foot Contact } \\
\text { Time (sec) }\end{array}$ & Normal & 209 & .23 & .07 & .22 & .766 & .682 & \\
\hline & Delayed & 42 & .23 & .08 & .21 & & & \\
\hline & Early & 49 & .50 & .12 & .51 & & & \\
\hline Step/Speed Rate & Normal & 209 & .51 & .09 & .50 & 2.221 & .329 & \\
\hline & Delayed & 42 & .49 & .09 & .48 & & & \\
\hline
\end{tabular}

*p $<0.05$

Analysis results in Table 9 a indicated significant difference among groups in terms of the body mass index, sitting height, leg length, stroke length, flexibility, left hand strength and right foot flight time parameters $(p<0.05)$. Girl students at a delayed maturation level had the lowest test values except the right foot flight time values, according to analysis results of a pairwise comparison. On the other hand, the pairwise comparison results among groups indicated that the test values of girl students at an early maturation level were higher than the test values of other groups except for the right foot flight time parameters. 
Table 8. Descriptive statistics of test parameters of girl students according to maturation levels

\begin{tabular}{|c|c|c|c|c|c|c|c|}
\hline & & \multicolumn{2}{|c|}{ Early $(n=36)$} & \multicolumn{2}{|c|}{ Normal $(n=174)$} & \multicolumn{2}{|c|}{ Delayed $(n=25)$} \\
\hline & & $\bar{x}$ & SD & $\bar{x}$ & SD & $\bar{x}$ & SD \\
\hline \multirow{8}{*}{ 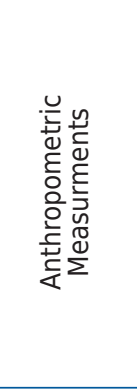 } & Age (year) & 8.16 & 2.13 & 8.12 & 2.11 & 8.08 & 2.59 \\
\hline & Height (cm) & 137.05 & 13.34 & 129.87 & 12.66 & 122.26 & 13.27 \\
\hline & Weight (kg) & 40.93 & 11.69 & 28.53 & 8.77 & 24.32 & 7.67 \\
\hline & Body Mass Index & 21.36 & 3.26 & 16.54 & 2.54 & 15.82 & 1.83 \\
\hline & Sitting Height $(\mathrm{cm})$ & 72.51 & 6.62 & 68.31 & 6.46 & 64.02 & 6.42 \\
\hline & Leg Length (cm) & 64.53 & 7.04 & 61.56 & 6.66 & 58.24 & 7.13 \\
\hline & Stroke Length $(\mathrm{cm})$ & 135.26 & 15.48 & 127.34 & 15.52 & 119.17 & 15.69 \\
\hline & Stroke Rate & -1.78 & 3.10 & -2.53 & 3.87 & -3.09 & 3.71 \\
\hline $\begin{array}{l}\text { Sit and } \\
\text { Reach }\end{array}$ & Flexibility $(\mathrm{cm})$ & 25.98 & 6.86 & 24.50 & 5.87 & 21.52 & 4.05 \\
\hline \multirow{2}{*}{$\begin{array}{l}\text { Hand Grip } \\
\text { Strength }\end{array}$} & Right Hand (kg) & 14.22 & 4.94 & 11.85 & 4.42 & 9.93 & 4.22 \\
\hline & Left Hand (kg) & 13.46 & 4.28 & 11.21 & 4.59 & 9.71 & 3.66 \\
\hline \multirow{2}{*}{$\begin{array}{l}\text { Vertical } \\
\text { Jump }\end{array}$} & Squat Jump (cm) & 11.86 & 4.03 & 13.56 & 4.16 & 12.99 & 4.02 \\
\hline & Countermovement Jump (cm) & 12.55 & 3.92 & 14.27 & 4.26 & 12.95 & 4.43 \\
\hline \multirow{3}{*}{$\begin{array}{l}\text { Postural } \\
\text { Sway }\end{array}$} & Anterior-Posterior Sway (mm) & 88.31 & 49.28 & 100.20 & 49.42 & 90.66 & 32.34 \\
\hline & Medial-Lateral Sway (mm) & 118.41 & 51.13 & 125.33 & 57.54 & 128.25 & 57.99 \\
\hline & Total Sway $(\mathrm{mm})$ & 163.10 & 74.81 & 177.96 & 75.05 & 176.00 & 72.50 \\
\hline \multirow{2}{*}{ Reaction } & Visual Reaction Time (sec) & 25.63 & 4.67 & 25.83 & 5.60 & 26.62 & 4.57 \\
\hline & Reaction Time (sec) & .81 & .10 & .82 & .14 & .78 & .13 \\
\hline Sprint & 20 Meters Sprint (sec) & 5.12 & .68 & 5.09 & .81 & 5.44 & 1.11 \\
\hline Agility & Pro-Agility Test Time (sec) & 7.53 & .99 & 7.37 & 1.09 & 7.88 & 1.57 \\
\hline \multirow{7}{*}{ 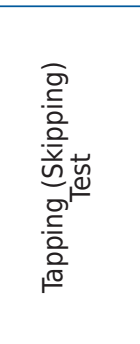 } & Right Foot Flight Time (sec) & .32 & .08 & .31 & .08 & .35 & .06 \\
\hline & Right Foot Contact Time (sec) & .24 & .09 & .21 & .05 & .22 & .06 \\
\hline & Right Foot Frequency (cycle/sec) & 2.23 & .65 & 2.24 & .55 & 1.89 & .34 \\
\hline & Left Foot Flight Time (sec) & .29 & .07 & .32 & .09 & .32 & .07 \\
\hline & Left Foot Contact Time (sec) & .24 & .06 & .22 & .06 & .22 & .05 \\
\hline & Left Foot Frequency (cycle/sec) & 2.21 & .49 & 2.15 & .50 & 2.04 & .44 \\
\hline & Step/Speed Rate & .51 & .09 & .48 & .10 & .54 & .08 \\
\hline
\end{tabular}

Table 9. One-way ANOVA analysis table of the parametric test values of girl students according to their maturation level

\begin{tabular}{|c|c|c|c|c|c|c|c|}
\hline & $\begin{array}{c}\text { Maturation } \\
\text { Level }\end{array}$ & $\mathrm{n}$ & $\bar{x}$ & SD & $\mathrm{F}$ & $p$ & $\begin{array}{l}\text { Significant } \\
\text { Difference }\end{array}$ \\
\hline \multirow{3}{*}{ Body Mass Index } & Early & 36 & 21.36 & 3.26 & \multirow{3}{*}{55.001} & \multirow{3}{*}{$.000 *$} & \multirow{3}{*}{$\begin{array}{l}\text { Early-Normal } \\
\text { Early-Delayed }\end{array}$} \\
\hline & Normal & 174 & 16.54 & 2.54 & & & \\
\hline & Delayed & 25 & 15.82 & 1.83 & & & \\
\hline \multirow{3}{*}{ Sitting Height (cm) } & Early & 36 & 72.51 & 6.62 & \multirow{3}{*}{12.914} & \multirow{3}{*}{$.000 *$} & \multirow{3}{*}{$\begin{array}{l}\text { Early-Normal } \\
\text { Early-Delayed } \\
\text { Normal-Delayed }\end{array}$} \\
\hline & Normal & 174 & 68.31 & 6.46 & & & \\
\hline & Delayed & 25 & 64.02 & 6.42 & & & \\
\hline \multirow{3}{*}{ Leg Length (cm) } & Early & 36 & 64.53 & 7.04 & \multirow{3}{*}{6.450} & \multirow{3}{*}{$.002 *$} & \multirow{3}{*}{$\begin{array}{l}\text { Early-Normal } \\
\text { Early-Delayed }\end{array}$} \\
\hline & Normal & 174 & 61.56 & 6.66 & & & \\
\hline & Delayed & 25 & 58.24 & 7.13 & & & \\
\hline \multirow{3}{*}{ Stroke Length $(\mathrm{cm})$} & Early & 36 & 135.26 & 15.48 & \multirow{3}{*}{8.089} & \multirow{3}{*}{$.000 *$} & \multirow{3}{*}{$\begin{array}{l}\text { Early-Normal } \\
\text { Early-Delayed } \\
\text { Normal-Delayed }\end{array}$} \\
\hline & Normal & 174 & 127.34 & 15.52 & & & \\
\hline & Delayed & 25 & 119.17 & 15.69 & & & \\
\hline
\end{tabular}


Table 9. continuation

\begin{tabular}{|c|c|c|c|c|c|c|c|}
\hline & $\begin{array}{l}\text { Maturation } \\
\text { Level }\end{array}$ & $\mathrm{n}$ & $\bar{x}$ & SD & $\mathrm{F}$ & $\mathrm{p}$ & $\begin{array}{l}\text { Significant } \\
\text { Difference }\end{array}$ \\
\hline \multirow{3}{*}{ Stroke Rate } & Early & 36 & -1.78 & 3.10 & & & \\
\hline & Normal & 174 & -2.53 & 3.87 & .978 & .378 & \\
\hline & Delayed & 25 & -3.09 & 3.71 & & & \\
\hline \multirow{3}{*}{ Flexibility (cm) } & Early & 36 & 25.98 & 6.86 & & & \multirow{3}{*}{$\begin{array}{l}\text { Early-Delayed } \\
\text { Normal-Delayed }\end{array}$} \\
\hline & Normal & 174 & 24.50 & 5.87 & 4.330 & $.014 *$ & \\
\hline & Delayed & 25 & 21.52 & 4.05 & & & \\
\hline \multirow{3}{*}{$\begin{array}{l}\text { Left Hand Strength } \\
(\mathrm{kg})\end{array}$} & Early & 36 & 13.46 & 4.28 & & & \multirow{3}{*}{$\begin{array}{l}\text { Early-Normal } \\
\text { Early-Delayed }\end{array}$} \\
\hline & Normal & 174 & 11.21 & 4.59 & 5.795 & $.004 *$ & \\
\hline & Delayed & 25 & 9.71 & 3.66 & & & \\
\hline \multirow{3}{*}{ Squat Jump (cm) } & Early & 36 & 11.86 & 4.03 & \multirow{3}{*}{2.581} & \multirow{3}{*}{.078} & \\
\hline & Normal & 174 & 13.56 & 4.16 & & & \\
\hline & Delayed & 25 & 12.99 & 4.02 & & & \\
\hline \multirow{3}{*}{$\begin{array}{l}\text { Countermovement } \\
\text { Jump }(\mathrm{cm})\end{array}$} & Early & 36 & 12.55 & 3.92 & \multirow{3}{*}{3.116} & \multirow{3}{*}{$.046 *$} & \\
\hline & Normal & 174 & 14.27 & 4.26 & & & \\
\hline & Delayed & 25 & 12.95 & 4.43 & & & \\
\hline \multirow{3}{*}{ Visual Reaction (sec) } & Early & 36 & 25.63 & 4.67 & \multirow{3}{*}{.288} & \multirow{3}{*}{.750} & \\
\hline & Normal & 174 & 25.83 & 5.60 & & & \\
\hline & Delayed & 25 & 26.62 & 4.57 & & & \\
\hline \multirow{3}{*}{ Reaction Time (sec) } & Early & 36 & .81 & .10 & \multirow{3}{*}{.806} & \multirow{3}{*}{.448} & \\
\hline & Normal & 174 & .82 & .14 & & & \\
\hline & Delayed & 25 & .78 & .13 & & & \\
\hline \multirow{3}{*}{$\begin{array}{l}\text { Right Foot Flight } \\
\text { Time (sec) }\end{array}$} & Early & 36 & .32 & .08 & \multirow{3}{*}{3.612} & \multirow{3}{*}{$.029 *$} & \multirow{3}{*}{$\begin{array}{l}\text { Normal- } \\
\text { Delayed }\end{array}$} \\
\hline & Normal & 174 & .31 & .08 & & & \\
\hline & Delayed & 25 & .35 & .06 & & & \\
\hline \multirow{3}{*}{$\begin{array}{l}\text { Left Foot Flight Time } \\
(\mathrm{sec})\end{array}$} & Early & 36 & .29 & .07 & \multirow{3}{*}{1.205} & \multirow{3}{*}{.302} & \\
\hline & Normal & 174 &, 32 &, 09 & & & \\
\hline & Delayed & 25 & ,32 &, 07 & & & \\
\hline
\end{tabular}

$*_{\mathrm{p}}<0.05$

According to the analysis results of non-parametric test values, there was a significant difference among groups with regard to test values of the right hand strength, anterior-posterior sway, right foot frequency and step/speed rate parameters $(\mathrm{p}<0.05)$. Girl students at an early maturation level had the highest right hand strength test values, and anterior-posterior sway values of the girl students at a normal maturation level were worse than test values of the girl students at an early and a delayed maturation level. Also, it was seen that the right foot frequency value of the girl students at an early and a normal maturation level was similar, and there was a significant difference between the right foot frequency values of normal and delayed maturation groups $(p<0.05)$. Although there was a significant difference among groups in terms of step/speed rate parameter ( $\mathrm{p}<0.05$ ), no significant difference among groups was determined according to the pairwise comparison of analysis results. 
Table 10. The Kruskal Wallis-H analysis table of the non-parametric test values of girl students according to their maturation level

\begin{tabular}{|c|c|c|c|c|c|c|c|c|}
\hline & $\begin{array}{l}\text { Maturation } \\
\text { Level }\end{array}$ & $\mathrm{n}$ & $\bar{x}$ & SD & Median & $\begin{array}{l}\text { Chi- } \\
\text { square }\end{array}$ & $p$ & $\begin{array}{l}\text { Significant } \\
\text { Difference }\end{array}$ \\
\hline \multirow{3}{*}{$\begin{array}{l}\text { Right Hand } \\
\text { Strength (kg) }\end{array}$} & Early & 36 & 14.22 & 4.94 & 13.25 & \multirow{3}{*}{13.736} & \multirow{3}{*}{$.001^{*}$} & \multirow{3}{*}{$\begin{array}{l}\text { Early-Normal } \\
\text { Early-Delayed }\end{array}$} \\
\hline & Normal & 174 & 11.85 & 4.42 & 10.80 & & & \\
\hline & Delayed & 25 & 9.93 & 4.22 & 8.20 & & & \\
\hline \multirow{3}{*}{$\begin{array}{l}\text { Anterior- } \\
\text { Posterior Sway } \\
(\mathrm{mm})\end{array}$} & Early & 36 & 88.31 & 49.28 & 77.61 & \multirow{3}{*}{6.781} & \multirow{3}{*}{$.034 *$} & \multirow{3}{*}{ Early-Normal } \\
\hline & Normal & 174 & 100.20 & 49.42 & 90.79 & & & \\
\hline & Delayed & 25 & 90.66 & 32.34 & 84.70 & & & \\
\hline \multirow{3}{*}{$\begin{array}{l}\text { Medial-Lateral } \\
\text { Sway (mm) }\end{array}$} & Early & 36 & 118.41 & 51.13 & 102.11 & \multirow{3}{*}{1.268} & \multirow{3}{*}{.530} & \\
\hline & Normal & 174 & 125.33 & 57.54 & 115.05 & & & \\
\hline & Delayed & 25 & 128.25 & 57.99 & 107.10 & & & \\
\hline \multirow{3}{*}{ Total Sway (mm) } & Early & 36 & 163.10 & 74.81 & 144.75 & \multirow{3}{*}{3.700} & \multirow{3}{*}{.157} & \\
\hline & Normal & 174 & 177.96 & 75.05 & 163.20 & & & \\
\hline & Delayed & 25 & 176.00 & 72.50 & 156.20 & & & \\
\hline \multirow{3}{*}{$\begin{array}{l}20 \text { Meters Sprint } \\
\text { (sec) }\end{array}$} & Early & 36 & 5.12 & .68 & 5.00 & \multirow{3}{*}{1.834} & \multirow{3}{*}{.400} & \\
\hline & Normal & 174 & 5.09 & .81 & 4.86 & & & \\
\hline & Delayed & 25 & 5.44 & 1.11 & 4.93 & & & \\
\hline \multirow{3}{*}{$\begin{array}{l}\text { Pro-Agility Test } \\
\text { Time (sec) }\end{array}$} & Early & 36 & 7.53 & .99 & 7.21 & \multirow{3}{*}{2.276} & \multirow{3}{*}{.320} & \\
\hline & Normal & 174 & 7.37 & 1.09 & 7.19 & & & \\
\hline & Delayed & 25 & 7.88 & 1.57 & 7.27 & & & \\
\hline \multirow{3}{*}{$\begin{array}{l}\text { Right Foot } \\
\text { Contact Time } \\
\text { (sec) }\end{array}$} & Early & 36 & .24 & .09 & .21 & \multirow{3}{*}{1.502} & \multirow{3}{*}{.472} & \\
\hline & Normal & 174 & .21 & .05 & .20 & & & \\
\hline & Delayed & 25 & .22 & .06 & .21 & & & \\
\hline \multirow{3}{*}{$\begin{array}{l}\text { Right Foot } \\
\text { Frequency } \\
\text { (cycle/sec) }\end{array}$} & Early & 36 & 2.23 & .65 & 2.11 & \multirow{3}{*}{9.872} & \multirow{3}{*}{$.007 *$} & \multirow{3}{*}{$\begin{array}{l}\text { Normal- } \\
\text { Delayed }\end{array}$} \\
\hline & Normal & 174 & 2.24 & .55 & 2.15 & & & \\
\hline & Delayed & 25 & 1.89 & .34 & 1.88 & & & \\
\hline \multirow{3}{*}{$\begin{array}{l}\text { Left Foot } \\
\text { Contact Time } \\
\text { (sec) }\end{array}$} & Early & 36 & .24 & .06 & .24 & & & \\
\hline & Normal & 174 & .22 & .06 & .20 & 5.953 & .051 & \\
\hline & Delayed & 25 & .22 & .05 & .20 & & & \\
\hline Left Foot & Early & 36 & 2.21 & .49 & 2.15 & & & \\
\hline Frequency & Normal & 174 & 2.15 & .50 & 2.11 & 2.022 & .364 & \\
\hline & Delayed & 25 & 2.04 & .44 & 1.86 & & & \\
\hline & Early & 36 & .51 & .09 & .51 & & & \\
\hline Step/Speed Rate & Normal & 174 & .48 & .10 & .49 & 6.131 & $.047^{*}$ & \\
\hline & Delayed & 25 & .54 & .08 & .50 & & & \\
\hline
\end{tabular}

$* \mathrm{p}<0.05$; the pairwise comparisons were examined by Mann Whitney-U test with Bonferroni correction.

\section{DISCUSSION}

The same performance parameters were compared in this study according to students' gender and maturation level. The significant difference was determined between boys and girls in terms of sitting height, stroke rate, flexibility, squat jump, reaction time, right foot flight time, body mass index, right hand strength, left hand strength, countermovement jump, anteriorposterior, medial-lateral and total sway, visual reaction, 20 meters sprint, proagility test time, right foot contact time, right foot frequency, left foot flight time, left foot frequency parameters $(\mathrm{p}<0.05)$. It was found that the sitting height, leg and stroke length, flexibility, right and left hand strength, anteriorposterior, medial-lateral and total sway, body mass index parameters of boy 
students significantly differed depending on the maturation level $(p<0.05)$. There was a significant difference among girl students of different maturation levels in terms of the body mass index, sitting height, leg and stroke length, flexibility, right and left hand strength, right foot flight time, anterior-posterior sway and right foot frequency parameters $(\mathrm{p}<0.05)$. Carvalho et al. [22] found a moderately positive correlation between the maturation offset and the knee extensor and flexor muscles isokinetic strength values of young male basketball players aged 14-16 years in eccentric and concentric exercises. It was revealed that the right and left hand strength values of boy students significantly differed depending on the maturation level in our study. The boy students at an early maturation level had higher hand strength values than boy students at other maturation levels, thus there was a similarity between the two studies.

A study of Wickel et al. [23] indicated that there was a significant difference between early maturation and other maturation groups with regard to leg length and body mass index values of girl students. The results of Wickel et al. [23] were similar to our study results in terms of the mentioned parameters. It may be said that the maturation level has a positive effect on anthropometric and physical performance parameters, and individuals at an early maturation level have an advantage in terms of exercise performance. The neuromuscular systems of individuals at an early maturation level may develop more than of those at a normal and a delayed maturation level, and this functional difference may affect physical performance. Similarly, Wickel and Eisenmann [24] determined a significant difference among early, normal and delayed maturation levels in both boy and girl students aged 13-14 years old in terms of leg length parameter. These authors also found that there was a significant difference among maturation groups in the body mass index values of boy students, and the body mass index values of early maturation group were higher than of other maturation groups. In the light of these findings, it could be said that early maturation was important for physical performance.

A study of Mahon et al. [25] indicated that the maturation offset and resting heart rate values significantly predicted maximal heart rate values. The maximal heart rate values during maximal exercise could be affected by the maturation level. The maximal heart rate values indicate the maximal loading rate of exercise, and individuals at an early maturation level could carry out the exercise efficiently. In our study, the right and left hand strength values of students at an early maturation level were higher than strength values of normal and delayed maturation groups. It could be said that the right and left hand strength values could be affected by the maturation level, such as the heart rate values determined in a study by Mahon et al. [25]. Gil et al. [26] reported that the maturity offset was a predictor of 30 meters velocity value with the total skinfold, fat-free mass, and chronological age. The fast muscle contraction is important for velocity exercises, and the muscle structure and physiology of individuals at an early maturation level may positively affect velocity performance. A significant difference was noted between the right foot frequency values of girl students at normal and delayed maturation levels in our study. The foot frequency values during the skipping test may be an indicator of speed and agility parameters. Although there was no significant difference between the agility test values of both boy and girl students in terms of the maturation level in our study, the difference in the right foot frequency values of girl students at normal and delayed maturation levels may be interpreted with regard to an agility parameter. 
Matthys et al. [27] reported that 20 meters sprint time and handgrip test values differentiated young handball players at early, normal and delayed maturation levels. Handball requires strong handgrip. In our study, higher hand strength values of students at an early maturation level support results of Matthys et al. [27]. Similarly, it was indicated that the maturity offset values of boys aged 1116 years correlated with speed, step length, step frequency and contact time parameters [28]. The maturation offset is an indirect indicator of individuals' maturation and growth. The findings of Meyers et al. [28] may be presented as evidence to the importance of the maturation level in physical activity and exercise performance. There was a significant difference between right foot frequency values of girl students from normal and delayed maturation groups in our study, which supported the step frequency-maturity offset relationship in findings of Meyers et al. [28].

Gay et al. [29] determined a significant correlation between the maturation offset and the body mass index, sitting height, leg length parameters. The body mass index, sitting height, leg length are important anthropometric parameters for many sport disciplines. The positive effect of the maturation level on anthropometric parameters in a study of Gay et al. [29] was supported by significant a difference among maturation level groups in terms of the sitting height, leg length and body mass index parameters found in our study. Gastin et al. [30] divided young male soccer players (10-18 years old) into five puberty stage groups and examined differences between the groups. The authors found that there was a significant difference between some puberty stage groups in terms of sitting height and 20 meters sprint values. The study results by Gastin et al. [30] also indicated that the puberty stage 4 and puberty stage 5 groups had better sitting height and sprint values than other puberty stage groups. Soccer players in late puberty may have better values of performance parameters because of an advanced level of physical maturation. The sitting height value of boy students at an early maturation level in our study was higher than values of other maturation groups. According to these findings, it may be said that the effect of the maturation level on the sitting height parameter is important. Santos et al. [31] analyzed horizontal jump, time of jump with single foot on 10 meters distance and tennis ball throw velocity values of adolescent individuals aged 11-17 years with and without using the maturation offset as a control variable. Their study indicated a significant difference between boy and girl adolescents in terms of performance parameters, with boys' performance parameters better than the girls. A significant difference was found in most performance parameters according to gender in our study, and these findings supported the study of Santos et al. [31]. Asadi et al. [32] also reported development in the values of vertical jump, peak power output, standing long jump, 20 meters sprint, 20 meters sprint with ball parameters of young male soccer players in the different maturation periods after six weeks' plyometric training period. Asadi et al. [32] reported the most development in 20-meter sprint and vertical jump parameters after six weeks' training in post-peak height velocity (post-maturation) group. There was no significant difference among maturation groups in terms of 20-meter sprint and vertical jump parameters of boy and girl students in our study. The difference between the two studies may arise from the conducting this study on boy and girl students in same age group.

Deliceoğlu [33] determined that there was a significant difference among maturation groups (early, normal, delayed maturation groups) in boys and girls aged 8, 9 and 10 years in terms of foot contact and flight time parameters. In his study, the jumping height values differentiated maturation groups in boys 
aged 6, 7, 8, 9 and 10 years and in girls aged 7, 8, 9 and 10 years. In our study, the girl students at a delayed maturation level had higher right foot flight time values than the normal maturation group. In Deliceoğlu's study [33], the foot flight time value of girls at an early maturation level was higher than in the normal and delayed maturation groups. The discrepancy between the findings of the two studies may be arise from individual differences in maturation of the study samples. In a study of Lätt et al. [34], there was a significant difference among maturation groups of boys aged 10-14 years in terms of fat-free mass and body mass index parameters, and it was determined that the values of boys at an early maturation level were higher than of boys at a normal and a delayed maturation level. The body mass index values of boy and girl students at an early maturation level in our study were the highest of all maturation groups, and this finding supported results of the study by Lätt et al. [34]. Segers et al. [35] indicated that the maximal oxygen uptake values at 8, 9.5 and $11 \mathrm{~km} / \mathrm{h}$ running speeds and thigh length values of male soccer players at an early maturation level were higher than in the delayed maturation group. In terms of differences of leg length values among maturation groups, the findings of our study showed a similarity to the study by Segers et al. [35]. Also, some anthropometric measurement values may affect the maximal oxygen uptake values, and the maturation level of boy and girl children predicting anthropometric parameters may be important in terms of aerobic endurance.

The study of Zago et al. [36] compared the sway values of male soccer players in different categories with regard to maturation levels and conditions of opened and closed eyes during the test. The authors observed a significant effect of the maturation level (categorization according to maturation offset) on the sway area, displacement velocity at center of pressure, anterior-posterior and mediallateral sway standard deviation parameters. In their study, soccer players with a high maturation offset had a lower postural sway standard deviation, which indicated an effect of the maturation level on postural sway parameters. The postural sway values of boy students at an early maturation level were lower than in other maturation groups in our study, and the anterior-posterior sway values of girl students at an early maturation level were lower than in other maturation groups in our study. Although both studies were conducted on children of different age groups, it could be said that their findings showed a similarity in terms of postural sway parameters. On the other hand, the male athletes aged 11-15 years were divided into three maturation groups (prePHV, mid-PHV, post-PHV groups) according to peak height velocity based the maturation offset in a study by Meylan et al. [37]. Their findings showed that the leg length and body mass index values of post-peak height velocity (postPHV) group with the highest maturation offset were higher than in groups of pre-peak height velocity (pre-PHV) and mid-peak height velocity (mid-PHV). In terms of the body mass index and leg length anthropometric parameters, the findings of our study supported the findings of Meylan et al. [37]. According to findings of these studies, it may be concluded that leg length and body mass index parameters are indicators of the maturation level, and these parameters may provide serious advantages to athletes during physical exercise.

A significant difference was seen between boy and girl students in terms of many measurement parameters in this study. The specific physiological structures of boys and girls may cause these differences of measurement parameters. In our study, there was no significant difference between the maturation groups in terms of some performance parameters. The maturation effect on some performance parameters may be lower than others, and the similarity in the maturation groups may be due to this situation. 


\section{CONCLUSIONS}

In conclusion, most anthropometric and motoric parameters of students aged 8 years differed depending on gender, and some parameters of both boy and girl students were affected by the maturation level factor. In the light of these findings, it may be concluded that the maturation level is an important factor in some anthropometric and motoric performance characteristics in boy and girl students aged 8 years, and the early maturation level positively affected these parameters. The maturation offset may be effectively used in exercises for children's athletic improvement. Future studies may be conducted on children and adolescents of different age groups, and the effects of the maturation level on physical fitness may be comprehensively revealed.

\section{REFERENCES}

[1] Malina RM, Rogol AD, Cumming SP, E.Silva MJC, Figueiredo AJ. Biological maturation of youth athletes: assessment and implications. Br J Sports Med. 2015; 49(13): 852-859. https://doi.org/10.1136/ bjsports-2015-094623

[2] Malina RM. Top 10 research questions related to growth and maturation of relevance to physical activity, performance, and fitness. Res Q Exerc Sport. 2014; 85(2): 157-173. https://doi.org/10.108 0/02701367.2014.897592

[3] Malina RM, Kozieł SM. Validation of maturity offset in a longitudinal sample of Polish boys. J Sport Sci. 2014; 32(5): 424-437. https://doi.org/10.1080/02640414.2013.828850

[4] Cumming SP, Sherar LB, Esliger DW, Riddoch CJ, Malina RM. Concurrent and prospective associations among biological maturation, and physical activity at 11 and 13 years of age. Scand J Med Sci Sport. 2014; 24(1): e20-e28. https://doi.org/10.1111/sms.12103

[5] Garcia C, Teles J, Barrigas C, Fragoso I. Health-related quality of life of Portuguese children and adolescents according to their biological maturation and volume of physical activity. Quality Life Res. 2018; 27(6): 1483-1492. https://doi.org/10.1007/s11136-018-1822-7

[6] Jakovljevic S, Macura M, Radivoj M, Jankovic N, Pajic Z, Erculj F. Biological maturity status and motor performance in fourteen-year-old basketball players. Int J Morphol. 2016; 34(2). https://doi. org/10.4067/S0717-95022016000200035

[7] Beunen G, Malina RM. Growth and biologic maturation: relevance to athletic performance. In: Hebestreit H, Bar-Or O, editors. The Young Athlete. Malden MA: Blackwell Publishing; 2008, 3-17. https://doi.org/10.1002/9780470696255.ch1

[8] Freitas DL, Lausen B, Maia JA, Lefevre J, Gouveia ÉR, Thomis M, Antunes AM, Claessens AL, Beunen G, Malina R. M.Skeletal maturation, fundamental motor skills and motor coordination in children 7-10 years. J Sport Sci. 2015; 33(9): 924-934. https://doi.org/10.1080/02640414.2014.977935

[9] Swain M, Kamper SJ, Maher CG, Broderick C, McKay D, Henschke N. Relationship between growth, maturation and musculoskeletal conditions in adolescents: a systematic review. Br J Sport Med. 2018; 52(19):1246-1252. https://doi.org/10.1136/bjsports-2017-098418

[10] Deprez D, Coutts AJ, Fransen J, et al. Relative age, biological maturation and anaerobic characteristics in elite youth soccer players. Int J Sport Med. 2013;34(10):897-903. https://doi. org/10.1055/s-0032-1333262

[11] Bundak R, Bas F, Furman A, et al. Sitting height and sitting height/height ratio references for Turkish children. Eur J Pediatr. 2014;173(7):861-869. https://doi.org/10.1007/s00431-013-2212-3

[12] Kılınç F. Effects of extremity height and girth on rowing performance in puberty period of children. Med J Süleyman Demirel Uni. 2008;15(3):30-33. https://doi.org/10.17343/sdutfd.93013

[13] Özer K. Antropometri, Sporda morfolojik planlama [Anthropometry, Morphological planning in sports]. Istanbul: Kazancı Matbaası; 1993. Turkish.

[14] Zorba E, Ziyagil MA. Vücut kompozisyonu ve ölçüm metotları [Body composition and measurement methods]. Trabzon: Erek Ofset; 1995. Turkish.

[15] Keys A, Fidanza F, Karvonen MJ, Kimura N, Taylor HL. Indices of relative weight and obesity. J Chronic Dis. 1972;25(6-7):329-343. https://doi.org/10.1016/0021-9681(72)90027-6

[16] Quetelet LAJ. A treatise on man and the development of his faculties. In: Wall R, editor. Comparative statistics in the 19th century. Farnborough, Hants: Gregg; 1973.

[17] Begatović E, Atiković A, Čuk I, Pajek MB. Influence of some basic motor abilities on the performance of bunny hop movement. Acta Kinesiologica. 2012;6(2):20-26.

[18] Kim Y, Todd T, Fujii T, Lim JC, Vrongistinos K, Jung T. Effects of Taekwondo intervention on balance in children with autism spectrum disorder. J Exerc Rehabil. 2016;12(4):314.

[19] Harman E, Garhammer J. Administration, scoring and interpretation of selected tests. In: Baechle TR, Earle RW, editors. Essentials of strength and conditioning. 3rd ed. Champaign, IL: Human Kinetics; 2008: 249-292.

[20] Malina RM, Bouchard C, Bar-Or O. Growth, maturation and physical activity. 2nd ed. Champaign, IL: Human Kinetics; 2004. 
[21] Mirwald RL, Baxter-Jones AD, Bailey DA, Beunen GP. An assessment of maturity from anthropometric measurements. Med Sci Sport Exerc. 2002;34(4):689-694. https://doi.org/10.1097/00005768200204000-00020

[22] Carvalho HM, Coelho-e-Silva M, Valente-dos-Santos J, Gonçalves RS, Philippaerts R, Malina R. Scaling lower-limb isokinetic strength for biological maturation and body size in adolescent basketball players. Eur J Appl Physiol. 2012;112(8):2881-2889. https://doi.org/10.1007/s00421-011-2259-7

[23] Wickel EE, Eisenmann JC, Welk GJ. Maturity-related variation in moderate-to-vigorous physical activity among 9-14 year olds. J Phys Activi Health. 2009;6(5):597-605. https://doi.org/10.1249/01. mss.0000322740.52753.79

[24] Wickel EE, Eisenmann JC. Maturity-related differences in physical activity among 13-to 14-year-old adolescents. Pediatr Exerc Sci. 2007;19(4):384-392. https://doi.org/10.1123/pes.19.4.384

[25] Mahon AD, Marjerrison AD, Lee JD, Woodruff ME, Hanna LE. Evaluating the prediction of maximal heart rate in children and adolescents. Res Q Exerc Sport. 2010;81(4):466-471. https://doi.org/10. 1080/02701367.2010.10599707

[26] Gil SM, Badiola A, Bidaurrazaga-Letona I, et al. Relationship between the relative age effect and anthropometry, maturity and performance in young soccer players. J Sport Sci. 2014;32(5):479-486. https://doi.org/10.1080/02640414.2013.832355

[27] Matthys SPJ, Vaeyens R, Coelho-e-Silva MJ, Lenoir M, Philippaerts R. The contribution of growth and maturation in the functional capacity and skill performance of male adolescent handball players. Int J Sport Med. 2012;33(07):543-549. https://doi.org/10.1055/s-0031-1298000

[28] Meyers RW, Oliver JL, Hughes MG, Lloyd RS, Cronin JB. Influence of age, maturity, and body size on the spatiotemporal determinants of maximal sprint speed in boys. J Strength Cond Res. 2017;31(4):10091016. https://doi.org/10.1519/JSC.0000000000001310

[29] Gay JL, Monsma EV, Smith AL, DeFreese JD, Torres-McGehee T. Assessment of growth and maturation in female athletes at a single point in time. Women in Sport and Physical Activity Journal, 2014; 22(2): 76-82. https://doi.org/10.1123/wspaj.2014-0018

[30] Gastin PB, Bennett G, Cook J. Biological maturity influences running performance in junior Australian football. J Sci Med Sport. 2013;16(2):140-145. https://doi.org/10.1016/j.jsams.2012.05.005

[31] Santos CF, Feitoza AHP, Ré AHN, Tudela MC, Cattuzzo MT, Henrique RDS. Effects of maturation as a covariate of sex difference on motor competence in adolescents. J Phys Educ. 2017;28. https://doi. org/ 10.4025/jphyseduc.v28i1.2830

[32] Asadi A, Ramirez-Campillo R, Arazi H, Sáez de Villarreal E. The effects of maturation on jumping ability and sprint adaptations to plyometric training in youth soccer players. J Sport Sci. 2018;36(21):24052411. https://doi.org/10.1080/02640414.2018.1459151

[33] Deliceoglu G. The jumping parameters of children according to maturity coefficient. The Anthropologist, 2016;23(3):367-377. https://doi.org/10.1080/09720073.2014.11891957

[34] Lätt E, Mäestu J, Rääsk T, Purge P, Jürimäe T, Jürimäe J. Maturity-related differences in moderate, vigorous, and moderate-to-vigorous physical activity in 10-14-year-old boys. Percept Motor Skills. 2015;120(2):659-670. https://doi.org/10.2466/10.PMS.120v13x0

[35] Segers V, De Clercq D, Janssens M, Bourgois J, Philippaerts R. Running economy in early and late maturing youth soccer players does not differ. Br J Sport Med. 2008;42(4):289-294. https://doi. org/10.1136/bjsm.2007.035915

[36] Zago M, Moorhead AP, Bertozzi F, Sforza C, Tarabini M, Galli M. Maturity offset affects standing postural control in youth male soccer players. J Biomech. 2020;99:109523. https://doi.org/ 10.1016/j. jbiomech.2019.109523

[37] Meylan CM, Cronin J, Hopkins WG, Oliver J. Adjustment of measures of strength and power in youth male athletes differing in body mass and maturation. Pediatr Exerc Sci. 2014;26(1):41-48. https:// doi.org/10.1123/pes.2013-0029 